\title{
Signal compression and enhancement using a new orthogonal-polynomial-based discrete transform
}

\begin{abstract}
Discrete orthogonal functions are important tools in digital signal processing. These functions received considerable attention in the last few decades. This study proposes a new set of orthogonal functions called discrete Krawtchouk-Tchebichef transform (DKTT). Two traditional orthogonal polynomials, namely, Krawtchouk and Tchebichef, are combined to form DKTT. The theoretical and mathematical frameworks of the proposed transform are provided. DKTT was tested using speech and image signals from a well-known database under clean and noisy environments. DKTT was applied in a speech enhancement algorithm to evaluate the efficient removal of noise from speech signal. The performance of DKTT was compared with that of standard transforms. Different types of distance (similarity index) and objective measures in terms of image quality, speech quality, and speech intelligibility assessments were used for comparison. Experimental tests show that DKTT exhibited remarkable achievements and excellent results in signal compression and speech enhancement. Therefore, DKTT can be considered as a new set of orthogonal functions for futuristic applications of signal processing.
\end{abstract}

Keyword: DKTT; Signal enhancement; Signal compression; Digital signal processing; Speech enhancement algorithm; Discrete Krawtchouk-Tchebichef transform; Orthogonal-polynomial-based discrete transform 